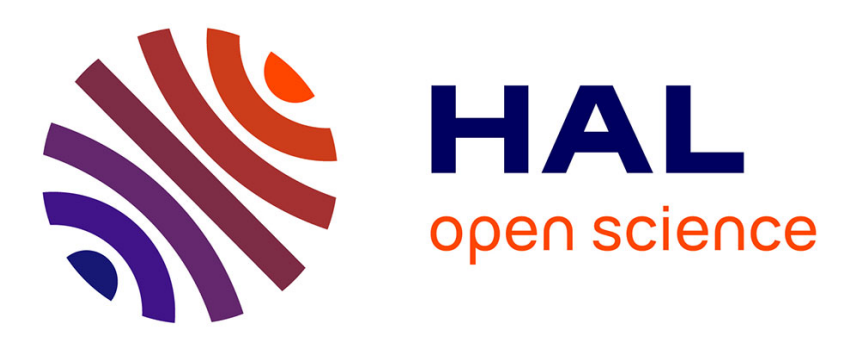

\title{
Structure et concept dans l'épistémologie mathématique de Jean Cavaillès
}

\author{
Hourya Benis Sinaceur
}

\section{To cite this version:}

Hourya Benis Sinaceur. Structure et concept dans l'épistémologie mathématique de Jean Cavaillès. Revue d'Histoire des Sciences, 1987, Mathématique et philosophie: Jean Cavaillès et Albert Lautman, 40 (1), pp.5-30. 10.3406/rhs.1987.4485 . halshs-01112688

\section{HAL Id: halshs-01112688 \\ https://shs.hal.science/halshs-01112688}

Submitted on 3 Feb 2015

HAL is a multi-disciplinary open access archive for the deposit and dissemination of scientific research documents, whether they are published or not. The documents may come from teaching and research institutions in France or abroad, or from public or private research centers.
L'archive ouverte pluridisciplinaire HAL, est destinée au dépôt et à la diffusion de documents scientifiques de niveau recherche, publiés ou non, émanant des établissements d'enseignement et de recherche français ou étrangers, des laboratoires publics ou privés. 


\section{Hourya Benis-Sinaceur}

\section{Structure et concept dans l'épistémologie mathématique de Jean Cavaillès}

In: Revue d'histoire des sciences. 1987, Tome 40 n¹. pp. 5-30.

\section{Citer ce document / Cite this document :}

Benis-Sinaceur Hourya. Structure et concept dans l'épistémologie mathématique de Jean Cavaillès. In: Revue d'histoire des sciences. 1987, Tome 40 n¹. pp. 5-30.

doi : $10.3406 /$ rhs. 1987.4485

http://www.persee.fr/web/revues/home/prescript/article/rhs_0151-4105_1987_num_40_1_4485 


\section{Résumé}

Résumé. - Le texte ci-après propose une interprétation de l'idée fameuse de Jean Cavaillès exhortant la philosophie de la science à devenir une « philosophie du concept ». Cette interprétation conjugue deux sources : 1) Les écrits de Cavaillès, y compris les fragments de lettres publiés par Gabrielle Ferrières dans son livre Jean Cavaillès, philosophe dans la guerre, et les lettres inédites à Albert Lautman, dont nous publions les plus significatives, selon nous, dans ce numéro de la Revue d'Histoire des Sciences. 2) Dans la mesure où nous avons pu le reconstituer, le contexte mathématique de ces écrits. Cette interprétation donne leur portée maximale à deux notions clés de l'épistémologie de Cavaillès : celles de "concept » et de "structure ", en observant qu'elles furent également au centre du développement de la mathématique dite " moderne ». Ce parallélisme mis à nu induit un rapport, naturel mais peu remarqué, entre la mathématique des structures, florissante en Allemagne à l'époque où Cavaillès s'y rendit et travailla (en particulier avec Emmy Noether), et la philosophie du concept qu'il proposa singulièrement dans une arène de pensée partagée, pour le moins, entre le néokantisme, la phénoménologie husserlienne et l'écoute heideggerienne de l'être.

\section{Abstract}

Summary. - In the text below I suggest an interpretation of Jean Cavaillès famous idea of urging the philosophy of science to become a "philosophy of concept ". This interpretation is based on two sources : 1) Cavaillès own writings, including parts of letters published by Gabrielle Ferrières in her book Jean Cavaillès, philosophe dans la guerre, and unpublished letters to Albert Lautman, of which we publish in this issue of Revue d'Histoire des Sciences the most revealing, in our opinion. 2) The mathematical context of these writings, insofar as we have been able to piece it together. This interpretation gives maximal import to two key notions of Cavaillès epistemology : those of " concept » and a structure ", because they played central roles in the development of what is called " modern » mathematics. This parallelism exposed here induces a natural, but little noticed relationship between the mathematics of structures, which flourished in Germany at the time that Cavaillès went there and worked (with Emmy Noether in particular), and the philosophy of concept, which he introduced in a peculiar way into an arena of thought that was, to say the least, divided namely, among NeoKantianism, HusserVs phenomenology and Heidegger's " awareness of being ».

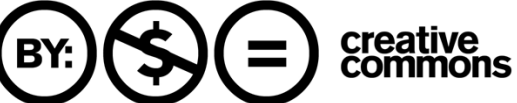




\title{
Structure et concept dans l'épistémologie mathématique de Jean Cavaillès*
}

\begin{abstract}
RESUMÉ. - Le texte ci-après propose une interprétation de l'idée fameuse de Jean Cavaillès exhortant la philosophie de la science à devenir une "philosophie du concept ". Cette interprétation conjugue deux sources : 1) Les écrits de Cavaillès, y compris les fragments de lettres publiés par Gabrielle Ferrières dans son livre Jean Cavaillès, philosophe dans la guerre, et les lettres inédites à Albert Lautman, dont nous publions les plus significatives, selon nous, dans ce numéro de la Revue d'Histoire des Sciences. 2) Dans la mesure où nous avons pu le reconstituer, le contexte mathématique de ces écrits. Cette interprétation donne leur portée maximale à deux notions clés de l'épistémologie de Cavaillès : celles de " concept " et de "structure ", en observant qu'elles furent également au centre du développement de la mathématique dite "moderne". Ce parallélisme mis à nu induit un rapport, naturel mais peu remarqué, entre la mathématique des structures, florissante en Allemagne à l'époque où Cavaillès s'y rendit et travailla (en particulier avec Emmy Noether), et la philosophie du concept qu'il proposa singulièrement dans une arène de pensée partagée, pour le moins, entre le néokantisme, la phénoménologie husserlienne et l'écou te heideggerienne de l'être.
\end{abstract}

SUMMARY. - In the text below I suggest an interpretation of Jean Cavaillès famous idea of urging the philosophy of science to become a "philosophy of concept". This interpretation is based on two sources : 1) Cavaillès own writings, including parts of letters published by Gabrielle Ferrières in her book Jean Cavaillès, philosophe dans la guerre, and unpublished letters to Albert Lautman, of which we publish in this issue of Revue d'Histoire des Sciences the most revealing, in our opinion. 2) The mathematical context of these writings, insofar as we have been able to piece it together. This interpretation gives maximal import to two key notions of Cavailles epistemology : those of " concept " and "structure ", because they played central roles in the development of what is called " modern" mathematics. This parallelism exposed

- Nous remercions très vivement Bartel Leender Van der Waerden pour avoir bien voulu lire les parties du texte relatives aux faits dont il fut acteur ou témoin. Certains passages doivent à ses remarques une meilleure formulation qu'ils n'avaient à l'origine.

Nous remercions chaleureusement Suzanne Lautman dont nons avons malheureusement appris le décès alors que nous corrigions cet article, de nous avoir aimablement communiqué les documents en sa possession, ci-après désignés par S. $L$. Nous la remercions également ainsi que Gabrielle Ferrières d'avoir autorisé la publication de ces documents.

Rev. Hist. Sci., 1987, XL/1 
here induces a natural, but little noticed relationship between the mathematics of structures, which flourished in Germany at the time that Cavaillès went there and worked (with Emmy Noether in particular), and the philosophy of concept, which he introduced in a peculiar way into an arena of thought that was, to say the least, divided namely, among Neo-Kantianism, Husserl's phenomenology and Heidegger's " awareness of being".

Il y a chez Jean Cavaillès une solidarité étroite entre l'étude de l'activité mathématique et une philosophie d'ordre plus général qu'il qualifie lui-même de " théorie de la raison ". Selon Méthode axiomatique et formalisme, la réflexion sur " l'essence du travail mathématique " conduit le philosophe à "creuser au-delà du mathématique proprement dit, dans le sol commun de toutes les activités rationnelles "(1), et l'autorise ainsi à se faire une idée du processus de la connaissance rationnelle en général. Pour Cavaillès les mathématiques, plus précisément la nouvelle mathématique abstraite qu'il voit à l'œuvre dans les travaux, encore récents à son époque, de Cantor, de Dedekind et de Hilbert, la " mathématique des algébristes" comme il l'appelle dans Méthode axiomatique et formalisme, est le modèle par excellence de l'activité de la raison. "La connaissance mathématique est centrale pour savoir ce qu'est la connaissance "(2). En effet, de toutes les disciplines de l'esprit, la mathématique est celle où la raison l'emporte si totalement sur les conditions de culture, l'essence sur les circonstances, la structure sur l'événement, qu'on attend rien moins de son étude qu'une doctrine de la pensée elle-même. La science, et, mieux que toutes les sciences, la mathématique, résulte " de ce caractère de la pensée de se développer selon son essence ", écrit Cavaillès en une formule suggestive (3). Expression d'une ferme conviction, qu'il va bientôt illustrer par une œuvre vouée à comprendre le " mécanisme des créations" mathématiques (4). La formation du savoir mathématique dans son procès effectif laisse voir la raison en exercice dans son activité la plus abstraite, la plus

(1) Méthode axiomatique ef formalisme (Paris : Hermann, 1981), 21. Les pages auxquelles renvoient nos citations appartiennent à cette deuxième ćdition. La première édition, également chez Hermann (Aclualités scientifques et industrielles, $\mathrm{n}^{\mathrm{os}} 608$ a 610$)$, date de 1938 .

(2) "La pensée mathématique „, compte rendu de la séance du 4 février 1939 , à la Société française de Philosophie, dans le Bulletin de la société, XI. (1946), 1-39, ici 34.

(3) Lettre à son père du 6 janvier 1928, dans G. Ferrières, Jean Cavaillès (Paris : PUF, 1950, rééd. 1982), 45 .

(4) Lettre du 22 avril 1928, ibid., 46-47. 
" pure ", celle dont les éléments externes sont justement rejetés dans une extériorité absolue parce qu'ils n'ont pas d'incidence sur son déploiement interne (s'ils en ont une sur son histoire).

\section{LE " NOUVEAU MONDE »}

La mathématique qui suscite la réflexion de Cavaillès, lui inspire les formulations les plus marquantes d'une doctrine attentive autant à la mobilité du savoir qu'à son essence, est la théorie abstraite des ensembles et la " mathématique des algébristes ". Si l'une vient de traverser, au moment où Cavaillès se met au travail après son agrégation de philosophie (1927), une grande crise, elle n'en a pas moins suscité une activité intense, aussi bien mathématique que logique ou métamathématique, sans parler des retombées épistémologico-philosophiques. Quant à l'autre, elle se trouve justement dans sa période de plein épanouissement et de belle assurance. Nous sommes en effet dans les années 20-30, qui ont vu Göttingen devenir le centre mathématique du monde. Le souvenir de Felix Klein (mort en 1925) est encore vivace. Hilbert est la figure la plus imposante, tandis que Emmy Noether, et bientôt Emil Artin à Hambourg constituent également des personnalités hautement attractives. "Quand j'arrivai à Göttingen en 1924, un nouveau monde s'ouvrit devant moi ", écrit par exemple B. L. Van der Waerden (5), qui n'allait pas tarder à retransmettre, de façon magistrale, le fruit de cette révélation.

Ce "nouveau monde ", Cavaillès le découvre aussi à sa façon. Boursier Rockefeller en 1929-1930, il séjourne successivement à Tübingen, Berlin, Hambourg, où il retrouve Herbrand, et Göttingen. L'année suivante, il est de nouveau à Hambourg, à Munich, à Fribourg où il prend contact avec Husserl. L'automne 1934 le voit à Berlin et à Göttingen; l'automne 1935 à Göttingen encore une fois ; l'automne 1936 à Hambourg et à Göttingen où il a toujours plaisir à revenir.

Ces incursions géographiques et intellectuelles sont déterminantes aussi bien pour ses positions philosophiques, qui passent par une explication serrée avec la phénoménologie, que pour sa réflexion sur les mathématiques, d'abord formée dans un climat

(5) On the sources of my book Moderne Algebra, Historia Mathematica, 2 (1975), 31-40. 
brunschvicgien. A la Staatsbibliothek de Hambourg, Cavaillès tombe sur un article de Adolph Fraenkel qui le remplit " d'une ardeur nouvelle "en lui révélant l'existence des correspondances de Georg Cantor (lettre du 17 novembre 1930) (6). Il forme alors le projet de dépouiller ces correspondances, car il espère non seulement y saisir l'apparition des difficultés de la théorie des ensembles, mais y vérifier aussi " le rôle déterminant et déterminé des autres branches des mathématiques ", de "ces échanges réciproques qui, à [son] avis, doivent suffire à expliquer le développement de la pensée mathématique comme un tout, sans l'intervention de la contingence historique " (7). On a, dès le départ, l'idée fondamentale de Cavaillès : l'étude historique d'un problème ou d'un ensemble de problèmes mathématiques aboutit à évacuer l'histoire de la formation des solutions ou de l'émergence de nouveaux problèmes (8).

Cavaillès ne dit rien sur le séminaire mathématique de l'Université de Hambourg, dont Otto Schreier (mort prématurément en 1929) et Emil Artin faisaient à cette époque un pôle d'attraction. Il ne semble pas avoir participé à l'effervescence que créait Artin autour de lui, ni succombé au charme de ses leçons qui eurent une grande influence sur plus d'un mathématicien. Göttingen, au contraire, est très présente dans les lettres à ses parents ou à Albert Lautman (9). Par ses institutions d'abord. L'institut de mathématiques, en effet, était un endroit " unique " (10), aux ressources remarquablement abondantes et accessibles. "Il m'a fallu une seconde pour trouver le livre dont j'avais envie ", écrira Cavaillès à ses parents, après leur avoir décrit l' "allégresse " avec laquelle il retrouve les

(6) Il s'agit de l'article de A. Fraenkel intitulé Georg Cantor, dans Jahresberichl der Deutschen Mathematikervereinigung, 39 (1930), 189-266. Cavaillès va le citer dans son avertissement à l'édition de la correspondance Cantor-Dedekind. Celle-ci, publiée en 1937 chez Hermann, est traduite en français dans le volume Philosophie mathématique (Paris : Hermann, 1962) qui reprend, par ailleurs les " Remarques sur la formation de la théorie abstraite des ensembles " parues une première fois en 1938 .

(7) Lettre du 17 novembre 1930, dans G. Ferrières, op. cil., 64-65.

(8) Cette idée, Cavaillès l'a exprimée maintes fois. Citons ce passage de la lettre inédite à A. Lautman du 5 mai 1937 : Rien n'est plus ennuyeux que l'histoire, surtout quand il faut la nier. "Documents S. L.

(9) En 1930, Göttingen était à son apogée. "Plus que jamais, on eût dit qu'un Congrès international de Mathèmaticiens se tenait perpétuellement dans la tranquille cité », écrit C. Reid, Hilbert (Berlin : Springer-Verlag, 1970), 191.

(10) C'est B. L. Van der Waerden qui le dit. " The mathematical library of Göttingen was unique. Everything one needed was there, and one could take the books from the shelves oneself ! In Amsterdam et in most continental universities this was impossible ", art. cit. n. 5, 33 . 
"petites rues de la ville", "souriantes dans la verdure ", et le " grand institut de mathématiques "(11) - l'édifice, inauguré en 1929, avait pu voir le jour grâce à l'action de Richard Courant, successeur de Felix Klein dans toutes les tâches d'organisation et de promotion des mathématiques, et à la générosité des Rockefeller (à qui on doit également, à la même époque, la construction de l'Institut Henri-Poincaré à Paris) (12). - Göttingen lui laissera des "souvenirs de bon travail ": il y étudie la correspondance Cantor-Dedekind avec l'aide et la collaboration de Emmy Noether. Celle-ci l'a accueilli " à bras ouverts ". Cavaillès évoquera, dans l'avertissement à l'édition des lettres Cantor-Dedekind, Emmy Noether et " ces journées de Göttingen, où il [lui] avait été donné après bien d'autres de connaître la bonté joyeuse de son accueil, l'intense rayonnement de son esprit " (13).

En 1930, lors du premier séjour de Cavaillès à Göttingen, Emmy Noether a déjà atteint la pleine maturité de son génie mathématique. Les grands mémoires sur la théorie des idéaux et la structure des anneaux (14) sont publiés. En 1921, Idealtheorie

(11) Lettre du 28 septembre 1934, dans G. Ferrières, op. cit., 97.

(12) L'histoire du séminaire mathématique de Göttingen peut être faite, pour les premières décennies du $\mathrm{xx}^{\mathbf{e}}$ siècle, à partir de deux livres de Constance Reid, Hilbert (Berlin : Springer-Verlag, 1970), et Courant in Göllingen and New York (ibid., 1976), et du livre édité par James W. Brewer et Martha K. Smith, Emmy Noether: A tribute to her life and work (New York/Basel : Marcel Dekker, 1981). Voir en particulier l'article de Saunders Mac Lane, "Mathematics at the University of Göttingen, 1931-1933 ", 65-78.

Pour Courant, Constance Reid souligne le rôle fondamental des années de formation comme élève, puis assistant, de Hilbert, et l'impact de l'exemple de Felix Klein dont il sut continuer l'action de promoteur d'une grande tradition scientifique.

Emigré aux Etats-Unis en 1934, Courant créera, à l'Université de New York, un nouvel Institut de Mathématiques qui porte aujourd'hui son nom. A la dernière page de son livre, Constance Reid se souvient des dernières paroles que lui dit Courant dans son bureau de l'Institut de New York, tandis qu'elle le complimentait sur l'esprit qu'il avait su y créer : "Ya, ya... It is Göttingen. Göttingen is here $n$ !

(13) Deuxième édition dans Jean Cavaillès, Philosophie malhémalique (Paris, Hermann : 1962), 185.

(14) On appelle anneau la structure algébrique constituée par la donnée d'un ensemble $A$ et de deux lois de composition appelées respectivement addition et multiplication et satisfaisant les axiomes suivants :

I Pour l'addition, $\mathrm{A}$ est un groupe commutatif (i.e. l'addition est associative, commutative, possède un élément neutre, et tout élément de A possède un opposé).

II La multiplication est associative et possède un élément neutre.

II L La multiplication est distributive par rapport à l'addition.

Si la multiplication est commutative, on dit que $\mathrm{A}$ est commutatif ou abélien. On dit qu'une partie $I \subset A$ est un idéal si I est un sous-groupe du groupe additif de $A$ et si les relations $a \in A, x \in I$ entrainent $a x \in I$ (pour simplifier, on a supposé A commutatif). 
in Ringbereichen (Mathematische Annalen, 83) définit les anneaux " noethériens " par la condition de chaîne ascendante (15) et généralise à ces anneaux le théorème que Lasker avait prouvé pour les idéaux de polynômes : dans un anneau noethérien tout idéal est intersection d'idéaux primaires. L'influence de ce travail sera si grande et si profonde qu'il apparaîtra après coup, et encore en 1970, comme une vraie "révolution " dans la théorie des idéaux (16). En 1926, Abstrakter Aufbau der Idealtheorie in algebraischen Zahl- und Funktionenkörpern (Math. Ann., 96) définit par cinq axiomes les anneaux appelés aujourd'hui " anneaux de Dedekind ", dans lesquels tout idéal est produit d'idéaux premiers et auxquels se généralise la théorie des idéaux d'un corps de nombres algébriques et d'un corps de fonctions algébriques d'une variable (17). L'influence de Emmy Noether est grande dans ces années 30. Grâce au cours que fait B. L. Van der Waerden, à Göttingen en 1927, sur la théorie des idéaux et qui eut un succès formidable, ses idées sont tombées dans le domaine public et ont rapidement gagné les grands centres mathématiques d'Europe. Bientôt, au Congrès international de Zürich (1932), elle obtient

(15) Il s'agit d'une condition de finitude : toute chaine ascendante d'idéaux est finie. Autrement dit, si $a_{1} \subseteq a_{2} \subseteq \ldots \subseteq a_{n} \subseteq \ldots$ est une telle chaine, alors il existe un entier $n$ tel que $a_{n}=a_{n+1}=a_{n+2} \ldots$

(16) Irving Kaplansky, Commutative Rings (Boston : Allyn \& Bacon, 1970), 5.

(17) Un idéal I d'un anneau $A$ est premier si les relations $a b \in I$ et $a \notin I$ entrainent $b \in \mathrm{I}$. Un idéal I est primaire si les relations $a b \in \mathrm{I}$ et $a \notin \mathrm{I}$ entraînent l'existence d'un nombre entier $k \geqslant 1$ tel que $b^{k} \in I$ (un idéal premier est évidemment primaire mais la réciproque est fausse).

Ces dénnitions permettent de schématiser la suite des étapes qui ont abouti à la définition abstraite et générale d'une structure étudiée par Dedekind dans le cas des corps de nombres algébriques.

Dedekind établit (Supplément XI aux Leçons de Dirichlet, 1894) que, dans un corps de nombres algébriques, tout idéal se décompose en produit d'idéaux premiers. L'analogie est parfaite avec la décomposition d'un nombre naturel en produit de nombres premiers.

En 1905, Lasker montre que pour les idéaux de polynômes, on a seulement la décomposition en PPCM (intersection) d'idéaux primaires (c'est lui qui définit la notion d'idéal primaire).

En 1921, Emmy Noether établit le théorème de décomposition d'un idéal en PPCM d'idéaux primaires dans tout anneau vérifiant la condition de chaîne ascendante. (L'expression a anneau noethérien " est due à Claude Chevalley, On the Theory of local Rings, Ann. of Malh., 44 ;1943), 690-708.)

En 1926, elle met en évidence les axiomes qui permettent d'aboutiı à la décomposition en produit d'idéaux premiers, et de retrouver l'analogie exacte avec la décomposition des nombres naturels. Ces axiomes définissent les " anneaux de Dedekind ". 
un véritable triomphe et la reconnaissance internationale de son œuvre. Le résumé qu'elle en fait (18) marque, par l'accueil qu'il suscite, le sommet de sa carrière scientifique.

\section{Le nouveau STyle mathématique}

2.1. A Göttingen, Cavaillès fait donc connaissance avec l'une des plus grandes figures de l'école algébrique allemande entre 1920 et 1933. L'autre grande figure de cette école qui " brillait d'un éclat exceptionnel "(19) était, on l'a dit, Emil Artin. Or, Emmy Noether et lui déterminent un nouveau style mathématique et, disons-le, une véritable doctrine qui imprime sa marque à la recherche non seulement en algèbre proprement dite, mais aussi en théorie des nombres ou en théorie des formes quadratiques ainsi que dans d'autres disciplines dont le développement s'accomplit alors dans sa mouvance : la géométrie algébrique, l'algèbre topologique et la topologie algébrique. P. S. Alexandroff, qui fut entre 1923 et 1933 très proche de Emmy Noether, reconnaît " l'influence essentielle " des idées de celle-ci "sur la pensée mathématique en général ". "Le développement des mathématiques aujourd'hui, affirme-t-il en 1935, est sous le signe de l'algébrisation, c'est-à-dire de la pénétration des idées et des méthodes algébriques dans les théories mathématiques les plus diverses "(20).

(18) Hyperkomplexe Systeme in ihren Beziehungen zur kommutativen Algebra und Zahlentheorie, Verhandl. Intern. Math.-Kongress Zurich, I (1932), 189-194. Traduction anglaise dans Emmy Noether : A tribute to her life and work (New York/Basel : James W. Brewer, Martha K. Smith, Marcel Dekker, 1981), 167-173.

(19) Jean Dieudonné, The work of Nicholas Bourbaki, Amer. Malh. Month., 77 (1970), 134-135.

(20) P. S. Alexandroff, a In Memory of Emmy Noether „, Address at the Moscow Mat. Soc., 5 septembre 1935. Reproduit dans Emmy Noether, Gesammelle Abhandlungen, hrsg. von Nathan Jacobson (Berlin : Springer-Verlag, 1983). Ce témoignage fut maintes fois corroboré. Henri Cartan, par exemple, évoquant les recherches au contact desquelles se formèrent les membres fondateurs du groupe Bourbaki, écrit : - Sous l'impulsion de l'école allemande (je pense surtout à la grande mathématicienne Emmy Noether, et à ses élèves), l'algèbre avait commencé à changer d'une façon essentielle toute la face des mathématiques ", Bourbaki und die heulige Mathematik, Arbeitsgemeinschaft für Forschung des Landes Nordrhein-Westfalen, Ht. 76 (Koln und Opladen : Westdeutscher-Verlag, 1959). - Voir aussi l'article plus récent de Saunders Mac Lane, History of abstract algebra : Origin, Rise, and Decline of a movement, dans Amer. Math. Herilage (Texas Tech University, Mathematics series, no 13, 1981), 3-35. 
Emil Artin, qui profita d'une occasion ultérieure (21) pour exposer explicitement son point de vue, voyait également en l'algèbre l' " instrument " des autres disciplines mathématiques, instrument de formulation ou de résolution de problèmes. N'en a-t-il pas, du reste, donné une brillante illustration en 1926-1927 dans la théorie algébrique des corps réels (22), dont ce n'était pas le moindre mérite que de rendre à la fois possible et (théoriquement) triviale la solution du dix-septième problème de Hilbert sur la décomposition des fonctions définies? (23).

Cette idée de la suprématie heuristique de l'algèbre est restée vivante longtemps après que Göttingen et Hambourg ont perdu le privilège de l'enseignement de Emmy Noether et de Emil Artin respectivement. Elle fut notamment défendue par un des plus marquants disciples de Artin : Claude Chevalley. Partenaire intellectuel de Cavaillès et proche de lui, son témoignage nous importe. Pour être de vingt ans postérieur aux années de travail de Cavaillès, il n'en est pas moins frappant par la netteté de son expression; et il nous montre simultanément la vivacité d'une tradition dont nous venons de situer le lieu et la date de naissance. En préface à son livre, Fundamental Concepts of Algebra (1956), Claude Chevalley écrit en effet :

"Algebra is not only a part of mathematics, it also plays within mathematics the role which mathematics itself played for a long time with respect to physics. What does the algebraist offer to other mathematicians ? Occasionally, the solution of a specific problem ; but mostly a language in which to express mathematical facts and a variety of

(21) "Contents and Methods of an Algebra Course „, Tata Institute (1960) ; reproduit dans The Collected Papers of Emil Artin (Reading, Mass : Addison-Wesley, 1965), 539-546.

(22) On appelle "réel " un corps dans lequel (-1) ne peut s'écrire sous la forme d'une somme de carrés d'éléments de ce corps. C'est là un critère, purement algébrique, caractérisant un type de structure porteur, par ailleurs, de propriétés topologiques. La théorie des corps réels fut élaborée au séminaire de Artin à Hambourg, avec la collaboration de $O$. Schreier, et la participation de B. L. Van der Waerden, ainsi que celui-ci nous l'a rapporté au cours d'un entretien personnel (8 décembre 1983). Sur l'importance et la fécondité de cette théorie, voir Ilourya Benis-Sinaceur, La constitution de l'algèbre réelle dans le mémoire d'Artin-Schreier, à paraître dans les Actes du Colloque d'Histoire des Mathématiques, Marseille-Luminy, juin 1983.

(23) On appelle définie une fonction de variables réelles qui n'a de valeur négative pour aucun système de valeurs de ses variables. En 1900, Hilbert avait demandé si toute fonction rationnelle, définie, est somme de carrés de fonctions rationnelles des mêmes variables. 
patterns of reasoning, put in a standard form. Algebra is not an end in itself : it has to listen to outside demands issued from various parts of mathematics."

2.2. En 1930, un homme. Bartel Leender Van der Waerden, " l'une des plus brillantes découvertes de l'école d'Emmy Noether" (24), et un livre, la Moderne Algebra, ont à la fois représenté ce point de vue et grandement contribué à le répandre. La Moderne Algebra réunit, en effet, et codifie les grands acquis de l'algèbre abstraite, y compris dans leurs développements les plus récents, dont les auditeurs des cours de Emil Artin ou de Emmy Noether pouvaient avoir connaissance. Ce livre met à la disposition de tous, retravaillés et complétés, ces cours que Van der Waerden a lui-même suivis avec enthousiasme : le cours d'algèbre, si "merveilleux ", fait par Artin à Hambourg au printemps 1926, et dont la matière se retrouve, en particulier, dans les chapitres de la Moderne Algebra consacrés à la théorie des corps et à la théorie de Galois (25); le séminaire sur la théorie des idéaux fait, l'hiver 1926-1927, par Artin, Otto Schreier, Wilhelm Blaschke et B. L. Van der Waerden lui-même; le séminaire où la théorie des corps réels, la fameuse "théorie de Artin-Schreier" vit le jour; le cours de Emmy Noether sur la " théorie des groupes et des nombres hypercomplexes"(1924-1925) dont le thème apparait également lors des discussions de Van der Waerden avec Artin et Schreier (26).

(24) C'est P. S. Alexandroff qui est l'auteur de ce trait.

(25) B. L. Van der Waerden a consigné de façon précise les sources de son livre dans l'article cité dans la note 5. Il y écrit : Artin " had promised to write a book on Algebra for the "yellow series" of Springer. We decided that I should take lectures notes and that we should write the book together - Courant, the editor on the series, agreed. Artin's lectures were marvellous. I worked out my notes and showed Artin one chapter after another. He was perfectly satisfed and said "Why don't you write the whole book?"”

(26) L'étude des systèmes de nombres hypercomplexes tient une bonne place dans les travaux de Emmy Noether et de Emil Artin. On appelait a systèmes de nombres hypercomplexes "ce que nous appelons aujourd'hui une "algèbre associative "sur un anneau A commutatif. E est une algèbre sur A (ou A-algèbre) si, et seulement si :

I E est un A-module;

II E est muni d'une multiplication interne distributive à gauche et à droite par rapport à l'addition et vérifiant la loi

$$
\alpha(x y)=(\alpha x) y=x(\alpha y) \quad \text { quels que soient } \alpha \in \mathrm{A} \text { et } x \in \mathrm{E}, y \in \mathrm{E} \text {. }
$$

Rappelons qu'un A-module est un ensemble muni :

1) d'une addition qui fait de $E$ un groupe commutatif; 
La publication de la Moderne Algebra, qui connaitra huit éditions et restera la référence privilégiée, sinon unique, jusque dans les années 70 , fut un véritable événement. Jean Dieudonné se souvient encore de l'espèce de choc qu'elle produisit sur ceux qui, n'ayant pas fréquenté les cercles de Hambourg ou de Göttingen, ignoraient l'existence de "l'algèbre moderne ". Il se souvient même du jour où l'ouvrage fut mis en vente pour la première fois, à Berlin où il se trouvait. " Je me précipitai, écrit-il, sur les deux volumes... et je fus stupéfait... Je ne savais pas ce qu'était un idéal, et à peine ce qu'était un groupe! " (27).

C'est dire le rôle du traité de Van der Waerden dans la diffusion, à l'échelle mondiale, des concepts et des méthodes de l'algèbre abstraite. De fait, non seulement ce traité rendait disponible un savoir neuf et encore peu répandu en dehors des frontières de l'Allemagne, mais encore il focalisait l'attention sur une nouvelle façon de faire de l'algèbre. La Moderne Algebra va éclipser le traité de Heinrich Weber et celui, antérieur, de Joseph Serret qui avaient successivement occupé la plus grande place sur le terrain didactique. Ainsi va-t-elle répercuter sur le plus large public de mathématiciens, en particulier ceux qui n'avaient pas eu un accès direct à l'enseignement des créateurs, les conceptions et les résultats indicatifs de la place centrale de l'algèbre dans les nouvelles mathématiques (28).

Le philosophe Albert Lautman, ami de Cavaillès et son partenaire dans une aventure philosophique portée par les résultats mathématiques récents, prend acte de ce fait dans son Essai sur

2) d'une multiplication externe vériflant :

$$
\begin{array}{ll}
\alpha(x+y)=\alpha x+\alpha y & \text { quels que soient } \alpha \in \mathbf{A} \text { et } x \in \mathbf{E}, y \in \mathbf{E} ; \\
(\alpha+\beta) x=\alpha x+\beta x & \text { quels que soient } \alpha \in \mathbf{A}, \beta \in \mathrm{A} \text { et } x \in \mathbf{E} ; \\
\alpha(\beta x)=(\alpha \beta) x & \text { quels que soient } \alpha \in \mathbf{A}, \beta \in \mathbf{A} \text { et } x \in \mathbf{E} ; \\
1 x=x \text { pour tout } x \in \mathbf{E} . &
\end{array}
$$

(27) * This treatise made a great impression. I still remember the day that Van der Waerden came out on sale... I rushed to those volumes and was stupefied... I did not know what an ideal was, and only just knew what a group was I (The work of Nicholas Bourbaki, Amer. Math. Month., 77 (1970), 134-135.) Dieudonné reconnaît que le traité de Van der Waerden fut un modèle pour la rédaction de celui de Bourbaki.

(28) G. Birkhoff écrit par exemple : "Van der Waerden made "Moderne Algebra" suddenly seem central in mathematics. It is not too much to say that the freshness and enthusiasm of his exposition electrified the mathematical world - especially mathematicians under 30 like myself ", dans Current Trends in Algebra, Amer. Math. Month., 80 (1973), 760-782; 81 (1974), 746. 
l'unité des sciences mathématiques dans leur développement actuel (29). Sa référence, comme en de multiples autres occasions, est le traité de B. L. Van der Waerden. Partant de la préface de Hermann Weyl à un de ses livres les plus célèbres (30), Lautman commente l'opposition de la mathématique nouvelle, "celle de la théorie des groupes et des algèbres abstraites ", à l'analyse du xıx e siècle ou "analyse de l'infiniment petit ". "En opposant les méthodes de la mathématique moderne à celles de la mathématique classique, écrit-il, Weyl pense, et les indications qu'il donne le prouvent, aux théories de l'algèbre moderne, telles qu'on les trouve exposées par exemple dans les deux tomes de l'ouvrage de M. Van der Waerden : Moderne Algebra (Berlin Springer, 1930). "Et Lautman se propose de faire voir comment ce conflit de méthodes "se dissipe au profit de l'algèbre ".

Bien entendu, Cavaillès connaissait (31) les travaux de Lautman (et réciproquement) (32). La lettre du 9 décembre 1937 indique qu'il a lu les thèses de son ami avant leur publication. Une fois publiées, il en a écrit un compte rendu pour la Revue de métaphysique et de morale (33). De la deuxième thèse, L'Essai sur l'unité des sciences mathématiques, il résume ainsi la teneur :

"M. Lautman montre avec rigueur que cet esprit de la mathématique moderne envahit non seulement l'algèbre et la topologie, mais aussi

(29) C'était sa thèse complémentaire pour le doctorat ès lettres. Soutenue en 1937, elle fut publiée, ainsi que sa thèse principale - et comme les thèses de Cavaillès -, dans la collection de leur maitre commun, Léon Brunschvicg, "Le Progrès de l'Esprit ", aux éditions Hermann, 1938, série des Actualites scientifiques et industrielles, no 589 à 591. Rédition récente dans Albert Lautman, Essai sur l'unilé des mathématiques et divers écrits (Paris : Union générale d'Editions, 1977).

(30) Il s'agissait de Gruppentheorie und Quantenmechanik (Leipzig : S. I Iirzel Verlag, $1928)$.

(31) Voir les lettres à A. Lautman du 3 septembre 1936, du 19 décembre 1936, du 9 décembre 1937, du 7 novembre 1938 (ci-après, p. 123-124), du 6 novembre 1942; Documents S. L.

(32) Voir, par exemple, la lettre à A. Lautman du 13 juin 1936, ci-après p. 120-121.

(33) T. 45 (1938). Supplément au numéro de juillet, 9-11. Ce compte rendu n'est pas signé. L'attribution à Cavaillès, dont nous sommes responsables, est permise par les lettres inédites à Lautman du 15 mai 1938 et du 17 mai 1938 (ci-après, p. 121 ). Documents S. I.

15 mai 1938: "Mon cher ami, voici le compte rendu de ta thèse - quoique à peine modifié je te l'envoie puisque c'était convenu, mais réexpédie le par retour du courrier. Que Parodi [secrétaire de rédaction de la Revue de mélaphysique el de morale] l'ait au début de la semaine.

17 mai 1938 : «... Il ressort de ta lettre que tu as transmis directement a Parodi mon compte rendu de tes thèses [souligné par Cavaillès]. Sinon avise-m'en aussitôt ici. o 
l'analyse, de sorte que l'unité des mathématiques serait rétablie par la pénétration des méthodes structurales et finitistes de l'algèbre au sein de l'analyse, et cela conformément aux idées de Hilbert. "

Ce texte n'implique évidemment pas que Cavaillès n'ait su de l'empire de l'algèbre abstraite que ce qu'en disait Lautman. Il est tout de même fort utile a posteriori; il confirme en toute clarté ce qu'un lecteur patient de l'œuvre de Cavaillès établit après de multiples recoupements.

Ainsi, lors des séjours à Göttingen et à Hambourg où Cavaillès va préparer les recherches qui aboutiront à la rédaction et à la publication en 1938 de ses deux thèses de doctorat, les " trois piliers " (34) de l'algèbre abstraite sont déjà solidement implantés, tant sur le plan didactique que sur le plan de la recherche. 'Théorie des groupes, en effet, théorie des corps et théorie des idéaux ont pris la forme de disciplines générales et abstraites et sont exposées avec maitrise et brio dans la Moderne Algebra. Emmy Noether, grand artisan du troisième pilier, accueille Cavaillès " à bras ouverts ", et collabore avec lui à l'édition de la correspondance Cantor-Dedekind. Cavaillès, qui démontre en 1932 quelques théorèmes sur les ensembles finis (Fundamenta Mathematicae, XIX, 143-148), n'aurait même pas eu besoin d'être un mathématicien créateur pour percevoir l'événement mathématique de son époque : le règne impérieux sinon exclusif de l'algèbre abstraite sur les autres branches.

\section{Deux manifestations du règne de l'algèbre abstratte}

Ce règne se traduit concrètement par deux phénomènes auxquels Cavaillès, s'il n'y contribue ou participe qu'indirectement, est en tout cas sensible ou attentif.

Le premier de ces phénomènes, nous l'avons déjà évoqué, est le rôle de leadership des séminaires de l'Université de Hambourg et de l'Université de Göttingen. Maître prestigieux, Artin attire nombre de mathématiciens de premier plan, parmi lesquels B. L. Van

(34) Van der Waerden, Die algebra seit Galois, Jahresbericht der Deutschen Mathematikervereinigung, 68 (1966). 
der Waerden, André Weil, Claude Chevalley, Jacques Herbrand, Max Zorn (35), Elie Cartan, etc. A. Weil, par exemple, raconte (36) que, dans les années 30 , tout jeune mathématicien désireux de se former aux idées nouvelles effectuait le pèlerinage de formation que représentaient un séjour à Hambourg et la fréquentation du séminaire de Artin. S'il ne semble pas qu'il ait suivi l'enseignement de Artin, Cavaillès a fait le pèlerinage de Hambourg.

B. L. Van der Waerden, de son côté, rappelle (37) qu'en dehors de David Hilbert, Felix Bernstein, Edmund Landau, Carl Runge, Richard Courant, Emmy Noether, qui en constituaient l'équipe permanente, en dehors des Privaldozenten comme Alexander Ostrowski, Helmut Kneser, Paul Bernays ou Otto Neugebauer, l'Institut de Mathématiques de Göttingen recevait des hôtes du monde entier : Hermann Weyl, John O. Neumann, Carl Siegel, Richard Brauer, Pavel S. Alexandroff, Kazimierz Kuratowski, Thoralf Skolem, Niels et Harald Bohr, Rolf Nevanlinna, Garett D. Birkhoff, Norbert Wiener et bien d'autres. Liste tout à fait impressionnante, surtout si on y ajoute encore les noms de Pavel S. Urysohn, de L. S. Pontryagin, de Alexander O. Gelfond, de Andrei N. Kolmogoroff, de Heinz Hopf, de Laslo Kálmár, de Oswald Veblen et de Solomon Lefschetz, tous présents à Göttingen à un moment où à un autre entre 1923 et 1928 (38). A Hambourg et à Göttingen,

(35) C'est à Hambourg et à cette époque que vit le jour le fameux a lemme de Zorn n. Dans une lettre à Paul J. Campbell, Zorn écrit : "I did my work on the Maximal Principle in Hamburg, around 1933. I proposed it as a "working principle" . as special case in algebra - It was well-received and became a "folk-affair". Artin would use it and Chevalley would take it up ", (P. J. Campbell, The origin of "Zorn's lemma ", Historia Mathematica, 5 : 1 (1978), 77-89).

(36) Entretien personnel, février 1982. De son côté, S. Mac Lane confirme qu'un séjour en Allemagne, à Göttingen de préférence, était chose nécessaire à la formation de tout mathématicien américain de cette époque. Sur 114 mathématiciens américains qui firent leur thèse à l'étranger, 34 la firent à Göttingen (Constance Reid, 1976, op. cit., n. 12, 129).

(37) Art. cit. n. 5.

(38) Cette liste montre évidemment que l'algèbre n'était pas l'unique discipline florissante aux séminaires de Göttingen ou de Hambourg, entre 1920 et 1933. Mais elle ne prouve pas que l'algèbre n'ait pas été alors une discipline dominante et souvent motrice. Nous avons déjà mentionné ci-dessus $(\$ 2)$ la part qu'elle eut dans le développement de la théorie des nombres, de la théorie des formes quadratiques ou de la topologie algébrique. Nous avons cité les témoignages de P. S. Alexandroff, de II. Cartan et de S. Mac Lane (cf. n. 20). Selon H. Weyl, le cercle des algébristes autour de Emmy Noether constitua en 1930 la partie la plus active de l'Institut de Göttingen (cf. n. 50). Cavaillès étant proche de certains membres fondateurs de Bourbaki, 
auréolées $\mathrm{du}$ prestige de mathématiciens si brillants, Cavaillès cherche donc sa propre voie.

Le deuxième phénomène, c'est l' "éclosion" de Nicolas Bourbaki, pour reprendre un mot d'Henri Cartan (39). Elle a lieu dès l'année 1934-1935 (40) et Cavaillès, sa lettre du 7 avril 1936 le prouve (41), est bien au fait de l'existence de l'entreprise du " traité collectif d'analyse ". Il sait que Claude Chevalley, avec qui il discute du dernier article logique de Jacques Herbrand, est chargé de la rédaction du fascicule de logique (42). A Strasbourg, en 1938, il côtoie amicalement Henri Cartan et André Weil, avec qui il discute de la collection d' "Essais philosophiques " qu'il veul fonder.

Selon une division alors dominante en Allemagne (43), le "traité collectif d'analyse "donne la première place aux structures algébriques et aux structures topologiques. Il est aussi fidèle à l'esprit d'abstraction de Artin et de Emmy Noether. Et, d'ailleurs, Henri Cartan croit bien " pouvoir affirmer " que, par l'influence qu'il exerça sur les jeunes chercheurs français des années 30, sur Claude Chevalley et André Weil en particulier, Artin a contribué " sans le savoir... à l'éclosion de Bourbaki ".

A l'exemple de Artin et de Noether, Bourbaki donne le beau rôle à la méthode axiomatique et met en avant l'idée de structure.

nous pouvons souligner une fois de plus la valorisation toute particulière de l'algèbre dans ce groupe. En 1975, Laurent Schwartz pouvait confier à Judith Friedmann-Miller : - Bourbaki m'a beaucoup algébrisé dans la mesure oú Bourbaki est beaucoup plus algébrique qu'analytique... (in Nicolas Bourbaki, Mémoire de l'EHESS, Paris, 1977). Enfin, n'oublions pas que Cavaillès a distingué " la mathématique des algébristes " comme une tendance propre à son époque et digne de ses réflexions.

(39) Emil Artin, Abh. malh. sem. Hamburg, 28 (1965), 1-6.

(40) H. Cartan, art. cit., n. 20.

(41) Dans G. Ferrières, op. cil., 107 : « Le groupe Bourbaki (Weil, Ehresmann, Henri Cartan, etc.) continue à m'envoyer la dactylographie de leur traité d'analyse, je le lis en ce moment $*$ lettre du 20 décembre 1938, ibid., 125.

(42) Ce fascicule de logique verra le jour en 1954 comme premier chapitre du Livre Premier ("Théorie des Ensembles") de la Première Partie ("Les structures Pondamentales de l'A nalyse $n$ ). Il est intitulé : Descriplion de la mathématique formelle.

(43) H. Weyl écrit en 1932 un article dont le titre est significatif : Topologie et algèbre abstraite, deux voies pour comprendre les mathématiques, Unterrichtsbläller für Malh. und Naturwiss., 38 (1932), 177-188. Reproduit dans Gesam. Abh., I I (Berlin : Springer-Verlag, 1968), 348-358. Voir aussi A half-century of Mathematics, Amer. Math. Month., 58, 523-553, dans Gesam. Abh., IV, 464-494. I.es deux pôles : algèbre, topologie, resteront majeurs pendant au moins deux décennies supplémentaires. Au premier Congrès international des Mathématiciens postérieur à la seconde guerre mondiale (Cambridge, Mass., 1950), trois communications sur quatre portent sur des thèmes en rapport avec l'algèbre abstraite ou la topologie. 
Et cela dès le début, c'est-à-dire dans ces années 1934-1935 où Cavaillès était si proche de certains de ses membres. "Ce qui nous a toujours guidés, raconte J. Dieudonné (44), c'est l'idée de structure... c'est absolument fondamental dans l'esprit de Bourbaki... dès le début..." (45).

\section{Structure et concept dans la mathématique " Moderne 》}

Il n'est pas utile de s'attarder sur l'importance bien connue des mathématiques structurales dont on vient de rappeler l'extraordinaire épanouissement dans l'école de Hilbert (à Göttingen et aussi à Hambourg) et dont Bourbaki fit son credo. Comme on sait, la pensée structuraliste a débordé, de loin, le champ mathématique. Mais, pour en rester aux mathématiques elles-mêmes, attirons l'attention sur un corollaire interne et immédiat de l'option structuraliste. Ce corollaire qui saute aux yeux dans les textes de Hilbert sur l'axiomatique, et peut-être davantage dans l'œuvre de Emmy Noether ou de Emil Artin, fut quelque peu émoussé dans l'héritage français bourbakiste. Mais Cavaillès eut un accès direct aux sources allemandes. Il sut que la mathématique structurale instituée par Hilbert est simultanément une mathématique conceptuelle.

Emil Artin et Emmy Noether, comme le grand maitre Hilbert, voient dans toute construction axiomatique une " construction de concepts ", une Begriffsbildung, en un sens, qui semble ne rien conserver cependant de son origine philosophique kantienne. Construire algébriquement la théorie des corps réels, par exemple, c'est ainsi construire des concepts, la fécondité de pareille entreprise étant démontrée par la solution du dix-septième problème de Hilbert.

Il est significatif, quand on sait le rapport de Van der Waerden à l'enseignement de Artin et de Noether, que ce terme de Begriffs-

(44) Entretien accordé à Judith Friedmann-Miller, reproduit dans son mémoire cité note 38 , p. 111 .

(45) Sur l'importance du concept de structure dans les mathématiques des années 30 , on peut encore citer, s'il en était besoin, le témoignage de Garett Birkhoff. Présent au Congrès de Topologie de Moscou, en 1935, il remarque : « La foi de certains jeunes congressistes dans l'approche abstraite "moderne" était évidente. Kurosh, par exemple, m'assura plus d'une fois avec insistance que "les Structures étaient très importantes". " Citation extraite de The Rise of Modern Algebra to 1936, Graduate Studies, Texas Tech University, 13 (october 1976), 61. 
bildungen ait été celui qui servit à caractériser la méthode de la Moderne Algebra. "In diese ganze Begriffswelt den Leser einzufuhren, soll das Hauptziel dieses Buches sein. "Introduire le lecteur dans ce monde du concept, tel était le but essentiel de Van der Waerden.

C'est que ces constructions de concepts sont " propres à devenir le support " de théories mathématiques nouvelles comme l'écrit Van der Waerden, en faisant l'éloge de Emmy Noether. Celle-ci ayant du reste partagé avec $J$. Herbrand et Claude Chevalley le privilège d'avoir compté dans la vie intellectuelle de Cavaillès, il est intéressant de rapporter ici le portrait que nous en a donné Van der Waerden :

"La maxime qui a constamment guidé Emmy Noether pourrait être formulée de la manière suivante : toutes les relations entre nombres, fonctions et opérations ne deviennent absolument claires, capables de généralisation et vraiment fécondes que lorsqu'elles sont libérées de leurs objets particuliers et réduites à des rapports généraux de concepts. Cette thèse (...) constituait un principe fondamental de sa pensée. Elle ne pouvait s'assimiler et retravailler aucun énoncé ni aucune démonstration avant de les avoir saisis abstraitement et rendus absolument clairs à l'œil de l'esprit. Elle ne pouvait penser que par concepts, non par formules, et en cela résidait sa force. Car elle était ainsi contrainte par sa propre façon d'être d'inventer les constructions de concepts propres à devenir le support des théories mathématiques" (46).

La begriffliche Mathematik, comme l'a baptisée P. S. Alexandroff, dont on peut faire remonter la tradition en Allemagne à Bernhard Bolzano, à Hermann Grassmann et à Richard Dedekind que Cavaillès a lus et commentés (47) apparait donc vers 1930 comme une véritable doctrine mathématique, source de lumière, certes, sur l'organisation et l'unité du savoir, mais source aussi de progrès. Après les travaux, alors tout récents, de Ernst Steinitz, de Emil Artin et de Emmy Noether, force était de reconnaitre l'extraordinaire fécondité de la méthode conceptuelle de Hilbert. Cavaillès a, du reste, particulièrement réfléchi sur cette méthode de Hilbert. Il a lu dans Axiomatisches Denken qu'une théorie

(46) Nachruf auf Emmy Noether, Mathematische Annalen, 111 (1935), 469-476. Reproduit dans le livre de A. Dick, Emmy Noether, 1882-1935 (Basel : Birkhäuser Verlag, 1970), et dans celui de J. Brewer et M. Smith cité dans la note 12.

(47) Méthode axiomalique et formalisme, 46-58. 
n'est rien d'autre que " l'établissement d'une certaine charpente de concepts "permettant une mise en ordre des faits (48). Et il sait les derniers succès de telles mises en ordre et de telles fabrications de concepts. Comme il l'écrit, " c'est l'essor de la théorie des corps en algèbre à la suite des travaux de Dedekind et de Steinitz. C'est en analyse la libération de ce "style des $\varepsilon$ ", dont parle Chevalley (...), c'est la théorie des espaces abstraits (...)" (49).

Il est vrai que Cavaillès a été très séduit par la problématique du fondement des mathématiques. Et que celle-ci est étrangère aux algébristes de 1930, qui sont des working mathematicians au sens strict, et qui, en tant que tels, ont totalement ignoré le rôle fondateur que Hilbert a cru, un moment, pouvoir conférer à l'axiomatique. Dans " la mathématique des algébristes ", l'axiomatique a exclusivement un rôle heuristique. Mutation dont Hermann Weyl a pris clairement acte en écrivant dès 1935 que la méthode pour "éclairer ou approfondir les fondements " s'était transformée en " instrument de la recherche mathématique concrète " (50). Mais Cavaillès, attentif par ailleurs à la position intuitionniste au point d'être allé à Rotterdam montrer sa thèse principale à Brouwer et à Heyting (51), n'a pas manqué de réserves vis-à-vis d'une position trop dogmatique pour reconnaitre la souplesse des processus effectifs de découverte, et pour laquelle " axiomatisation et formalisation ne sont plus moments d'une dialectique créatrice mais des uniformes obligatoires " (52). Il va même jusqu'à rendre ce dogmatisme responsable de l' " exagération " dans les difficultés de la théorie des ensembles : " réellement, il n'y a, dit-il, que celles qui proviennent, dı mélange entre spéculation philosophique et raisonnements mathématiques et celles, normales, que provoquent les insuffisances techniques ".

"Moment d'une dialectique créatrice ", "instrument de recherche concrète ", l'affirmation de Cavaillès fait écho à celle de Weyl, et la position dernière de Cavaillès sur l'axiomatique n'est pas loin de convenir à la pratique de son époque telle qu'il pouvait en faire l'expérience auprès de Emmy Noether.

(48) Ibid., 77.

(49) Ibid., 78.

(50) Emmy Noether, Memorial Adress n, Bryn Mawr College, 26 avril 1935. Reproduit dans H. Weyl, Gesam. Abh., II I, 425-444.

(51) G. Ferrières, op. cil., 116.

(52) Méthode axiomalique el formalisme, 182. 


\section{LA Philosophie de L'algèbre abstraite}

5.1. Il est difficile de mesurer de façon précise la résonance sur la réflexion épistémologique de Cavaillès de la mathématique des algébristes, à laquelle il s'est initié par la lecture de Dedekind. Dans les écrits de Cavaillès, les références directes aux travaux mathématiques sont rares. Le matériau n'affleure jamais à nu mais déjà élaboré et complètement refondu dans les interrogations proprement philosophiques qui encadrent ou orientent la réflexion sans l'enfermer dans des catégories préétablies.

$\mathrm{Si}$ on examine ses sources d'une façon objective et extérieure, on s'aperçoit qu'il connaît et utilise les œuvres de Dedekind et de Hilbert. Outre les opuscules sur les nombres entiers et les nombres réels de Dedekind, il cite (53) les œuvres complètes éditées de 1930 à 1932 par Oysten Ore, Robert Fricke et Emmy Noether. De Hilbert, outre les Gundlagen der Geometrie, Uber den Zahlbegriff et la fameuse conférence au II e Congrès international des Mathématiciens (Paris, 1900), il cite tous les articles de métamathématique qui débattent, d'un point de vue autre que celui de la stricte efficacité mathématique, du rôle de l'axiomatique, des fondements de la logique et de l'arithmétique ou des mathématiques en général, de l'infini, etc. A la séance du 4 février 1939 de la Société française de Philosophie, Cavaillès reconnait avoir décrit, dans sa thèse sur la méthode axiomatique, quelques-uns des procédés mathématiques en s'inspirant à la fois de Hilbert et de Dedekind (54). Il renvoie à la leçon d'habilitation prononcée par Dedekind le 30 juin 1854, en présence de Gauss et publiée pour la première fois en 1932 par Emmy Noether (55). La pensée de Cavaillès entretient un rapport essentiel avec les idées développées dans cette leçon. On y trouve, en effet, affirmée et illustrée la " nécessité interne " qui modèle avec une force "contraignante " (innere, zwingende Notwendigkeit) le développement des mathématiques (et de toutes les autres sciences).

En ce qui concerne les "algébristes " plus récents ou même contemporains, on ne peut affirmer qu'il ait une connaissance directe de leurs travaux. Il est informé, cependant, du développement

(53) Bibliographies de ses deux thèses.

(54) Bulletin de la Société française de Philosophie, XL (1946), 10.

(55) R. Dedekind, Gesammelle mathematische Werke, II I (Braunschweig, 1930-1932), $428-438$. 
de la théorie des corps. Nous avons cité plus haut un des passages de Méthode axiomatique et formalisme qui invoque Steinitz. Il y en a un autre (p. 86) qui laisse penser que c'est par Emmy Noether que Cavaillès a entendu parler de Steinitz et du fait que la théorie des corps algébriquement clos (caractéristique non précisée) admet " une infinité d'interprétations non isomorphes" (56). Il est vraisemblable, par ailleurs, que Cavaillès a lu l'article de Helmut Hasse, "Die moderne algebraische Methode" (1930), qui fait partie du même volume 39 du Jahresbericht der Deutschen Mathematiker Vereinigung où il a trouvé, tandis qu'il était à Hambourg, le Georg Cantor de A. Fraenkel, si volumineux qu'il a craint d'y voir sa thèse déflorée, si précieux par l'indication de la correspondance inédite Dedekind-Cantor. Ce volume 39 du Jahresbericht était, au moment où Cavaillès le lit, la " dernière livraison " de la revue. Sa lettre laisse comprendre qu'il est tombé dessus tout à fait par hasard et, comme souvent dans ces cas-là, il a bien dû parcourir l'ensemble du volume. Or Helmut Hasse insiste, dans son article, sur le caractère du formalisme de l'algèbre qui est, non pas un jeu de formules vides, mais " une délimitation par formules logiques ou mathématiques précises de son contenu conceptuel "(57). Et ce contenu conceptuel est illustré par les exemples essentiels de la construction algébrique des corps de Steinitz - que Hasse a réédité en fascicule séparé, précisément cette année 1930 - par la construction algébrique des corps réels de Artin et Schreier, et par la théorie abstraite des idéaux de Emmy Noether. Si l'on ajoute les travaux de Alexander Ostrowski et de Wolfgang Krull dont il résume également l'essentiel, on peut dire que Hasse faisait un panorama des principaux acquis de l'algèbre abstraite vers $1930(58)$.

(56) Cavaillès invoque l'exemple des a corps algébriquement fermés absolus de Steinitz " parmi d'autres exemples illustrant l'idée d'une théorie non calégorique, c'est-à-dire n'ayant pas un modèle unique à isomorphisme près. Rappelons qu'en 1934 Thoralf Skolem avait montré que la théorie de l'arithmétique des entiers est non catégorique. Cavaillès s'intéresse à la notion de catégoricité, ainsi qu'à celles d'indépendance et de saturation dans le cadre d'une analyse de la méthode axiomatique qui occupe le chapitre II de son livre.

(57) Souligné par l'auteur.

(58) Signalons également la publication, en 1936, d'un fascicule sur I.'Algèbre abstraite dans la série Actualités scientifiques el industrielles des Editions Hermann, avec lesquelles Cavaillès négociera la direction d'une collection d' a Essais philosophiques 2. Ce fascicule, no 362 , dù à Oysten Ore, présente un tableau synthétique et élémentaire des principaux résultats de l'algèbre abstraite. 
En tout état de cause, c'est au développement de l'algèbre abstraite, autant qu'à Husserl, que Cavaillès emprunte les procédés distingués l'un comme " thématisation" (59), l'autre comme "idéalisation " (60), et qui deviendront, dans Sur la logique et la théorie de la science, des "propriétés constitutives de l'essence de la pensée "(p. 27).

Les pages sur le "paradigme", dans cet ouvrage (Paris, PUF, 1960, p. 27-30), demeureraient fort difficiles si on n'y reconnaissait les effets de l'adjonction d'éléments idéaux : suppression de singularité, dissociation entre objet particulier de la première intuition et objet général, " dissociation libératrice de sens ", source de pluralité dans l'unité formelle et origine de "ces promotions mathématiques " successives auxquelles nous devons chaque fois de nouveaux objets. C'est le vertige de la créalion qu'atteste la polymorphie fusant d'un " unique enchaînement rationnel ", et se donnant, sur un autre registre, comme "fuite indéfinie vers le sens qui, dégagé grâce au décor intelligible et de lui fabriqué, s'échappe aussitôt "comme dans un rêve" et par là même pose son originalité ". L'idéalisation c'est le passage, nécessairement progressif, étalé sur une certaine "longueur d'enchaînement ", de l'acte - additionner des nombres entiers, par exemple - au sens - additionner sans spécification d'individus de la "liaison-acte " à la "liaison-type ". D'où son autre nom "paradigme".

La " thématisation ", elle, " prend pour départ l'enchaînement saisi cette fois dans son vol, trajectoire qui se mue en sens. La pensée ne va plus vers le terme créé mais part de la façon de créer pour en donner le principe par une abstraction de même nature que l'autre, mais dirigée transversalement "(p. 30). Ce deuxième processus est conditionné par le premier. Car, dans le passage de l'acte au sens apparaît une dualité : entre le sens d'une opération en tant qu'elle est opérée et son sens en tant qu'opérante. Ce n'est

(59) Dans la ligne de II usserl, Cavaillès appelle "thématisation "le fait que "les gestes accomplis sur un modèle ou un champ d'individus peuvent, à leur tour, être considérés comme des individus sur lesquels le mathématicien travaille en les considérant comme un nouveau champ * (compte rendu de la séance du 4 février 1939 de la Société française de Philosophie, 10). Noter en passant l'emprunt du terme "champ • à la thèse de II erbrand.

(60) L' "idéalisation" "consiste simplement à exiger qu'une opération, qui se trouvait d'une manière accidentelle limitée à certaines circonstances extrinsèques à l'accomplissement même de cette opération, soit libérée de cette limitation extrinsèque, et ceci par la position d'un système d'objets qui ne coïncide plus avec les objets de l'intuition. C'est par exemple ainsi que sont faites les différentes généralisations de la notion de nombre " (op. cil., n. 59, ibid.). 
plus seulement ce passage de l'acte au sens qui retient l'intérêt, mais le comment de ce passage; non plus le résultat en tant que résultat, si général soit-il devenu, mais les modes de sa génération. Et pour reprendre un exemple simple, non plus l'addition indifférente aux nombres, l'addition abstraite livrée par le procès longitudinal d'idéalisation, mais les lois d'associativité et de commutativité. Ou, pour invoquer l'histoire de la logique, non plus écrire le système de tous les formalismes possibles - solution que Cavaillès attribue aussi bien à Frege qu'à Dedekind (p. 33) 一, mais dresser l'ensemble des syntaxes de tous les systèmes formels projet commun, selon Cavaillès, à Carnap et à Tarski.

Il y aurait encore bien d'autres finesses à relever dans l'enchevêtrement de ces deux processus de l'algèbre ou de la pensée. Nous voulions surtout assigner le champ particulier par référence auquel Cavaillès en a forgé la définition et amorcé la généralisation.

5.2. La vision générale qu'avait Cavaillès des mathématiques comme totalité organique et douée de spontanéité n'est assurément pas sans rapport avec la conception imposée par l'axiomatique d'une science vigoureusement charpentée et unitaire dans la "prolifération exubérante " de ses résultats. Si on se souvient que Hilbert écrivait dès 1900 (61) que la " science mathématique est un entier indivisible, un organisme (62) dont la force vitale a pour condition l'indissolubilité de ses parties " et que "plus une théorie mathématique se développe..., plus on découvre des relations entre cette théorie et des branches qui lui étaient étrangères jusque-là ", on comprend mieux pourquoi Cavaillès avait tellement à cœur, dès le départ, d'expliquer le développement de la pensée mathématique " de façon strictement intrinsèque par les échanges réciproques " des diverses disciplines mathématiques, sans l'intervention de la contingence historique et sans le branle d'une impulsion extérieure. En devenant abstraite l'axiomatique renforce encore cette impression d'un dynamisme autonome et unificateur. Cavaillès a perçu l'effet proprement moteur de l'abstraction et qu'en schématisant ce sont de nouvelles dépendances qu'elle révèle, des liens parfois inattendus, une solidarité ramifiée mais plus forte de l'ensemble.

(61) Conférence au I I e Congrès international des Mathématiciens.

(62) Cette métaphore biologique est également présente dans la leçon de Dedekind citée par Cavaillès, Gesam. malh. Werke, III, 430. 
Bien avant que les mathématiciens du groupe Bourbaki n'aient eu le temps de rendre ces idées familières au public français, Cavaillès en avait tiré la leçon quant aux réquisits d'une épistémologie travaillée de l'intérieur par le contenu et le mouvement effectif de la science à laquelle elle s'applique. Aussi n'est-ce pas un hasard qu'y émergent les notions dont elle fait une double pierre angulaire, de structure et de concept.

\section{Structure et CONCEPT DANS LA PHILOSOPHIE \\ DE L'ALGÈBRE ABSTRAITE}

6.1. La notion de structure a une place considérable dans l'épistémologie de Cavaillès. D'abord la structure préside à l'organisation du savoir. La théorie de la science est théorie de la structure de la science. Bolzano, en qui Cavaillès reconnait après Husserl un précurseur, l'avait compris. Avec Bolzano, " pour la première fois peut-être, la science n'est plus considérée comme simple intermédiaire entre l'esprit humain et l'être en soi, dépendant autant de l'un que de l'autre et n'ayant pas de réalité propre, mais comme un objet sui generis, original dans son essence, autonome dans son mouvement "(63). D'où l'intérêt pour la démonstration, car " la science est avant tout théorie démontrée " et " la structure de la science non seulement est démonstration, mais se confond avec la démonstration ". La démonstration crée ces chaînes de dépendances qui font des mathématiques, au sens propre, un tissu de nécessités.

Ensuite, la structure commande le progrès de la science. Car l'enchainement déductif est " essentiellement créateur des contenus qu'il atteint " (64). La science effective est " science militante " (65). "La démonstration, par cela seul qu'elle pose le but, étend et ramifie le domaine créé au moyen de combinaisons qu'elle établit aussitôt qu'elles sont possibles " (66). La structure est donc principe de mouvement, de progrès. C'est en vertu de leur structure que méthodes et théories ont cette indépendance relative qui provoque

(63) Sur la logique et la théorie de la science (Paris : puF, 1960), 21. Nous n'avons envisagé ici cet ouvrage que dans les aspects relatifs à la question déterminée qui nous occupe. Pour une vue plus globale de l'épistémologie mathématique de Cavaillès, nous renvoyons à un travail antérieur publié dans Critique, no 461 (octobre 1985).

(64) Sur la logique et la théorie de la science (Paris : PU F, 1960), 73.

(65) Ibid., 23.

(66) Ibid., 25. 
les rencontres de plusieurs disciplines et les renversements d'orientation, parfois imprévisibles (résultats de Gödel qui établissent la complétude du calcul des prédicats du premier ordre et l'incomplétude de la plus " simple " des sciences mathématiques, l'arithmétique). Cavaillès est d'accord avec les tenants de l'axiomatique abstraite : axiomatiser n'est pas répondre au vœu d'un formalisme vide et stérile (67), mais véritablement inventer. S'il est vrai, comme l'a rapporté Alexandroff, que toute la personnalité de Emmy Noether était opposée à la tendance, alors à l'honneur dans certains cercles de mathématiciens, qui ne voyaient dans l'axiomatique qu'un jeu formel, Cavaillès a été à même d'en témoigner. L'axiomatisation rend visibles les structures générales impliquées dans les problèmes particuliers. La généralité - fille de l'idéalisation - n'est pas recherchée pour elle-même, mais par souci d'efficacité. Les structures - repérées par thématisation - ont pour corrélats des concepts abstraits : corps, anneau, idéal, algèbre. Et les concepts abstraits sont porteurs de nouveauté : on l'a éprouvé dans la création des corps " réels" par Artin et Schreier, dans la définition des anneaux noethériens ou des anneaux artiniens (68). L'épistémologie de Cavaillès, qui soustrait la science autant à l'immobilité des êtres en soi qu'à " l'univers des objets de culture " "signification en dehors de leur existence actuelle (et déterminée) ", autant aux pièges de la catégorialisation ontologique (Kant) ou logique (Carnap) qu'à ceux d'une formalisation totale (Frege), aménage, certes, une voie extrêmement étroite. L'effectif y est nécessaire et le progrès s'enracine dans les formes du nécessaire, accompli ou manifesté dans l'exigence de solution des problèmes. Cette épistémologie rend compte, toutefois, du mouvement réel par lequel s'est traduite l'efficacité créatrice de la constitution des structures abstraites. Comme il l'écrit à Albert Lautman le 13 juin 1936 (69), Cavaillès reconnait d'ailleurs l'impact sur sa pensée de ce mouvement des structures : "Sous l'influence

(67) Voir l'article posthume, publiè par les soins de Georges Canguilhem et de Jean Lameere, dans la Revue internationale de Philosophie, no 8 (avril 1949). Tenant compte du théorème d'incomplétude de Gơdel, Cavaillès y examine les difficultés d'une conception des mathématiques comme pure combinatoire.

(68) On appelle a artinien " un anneau vériflant une condition duale de celle définie ci-dessus dans la note 15. Artin utilisa systématiquement cette condition dite de - chaîne descendante "considérée par Emmy Noether dans son mémoire de 1921.

(69) Ci-après, p. 120-121. 
du langage qui me submerge, je suis pris par des images architecturales assez réalistes du développement mathématique..."

6.2. Si la mathématique des structures abstraites est toujours aussi une mathématique de concepts comme en témoignent, dans les années 30, Hilbert, Artin, Noether, Van der Waerden, il était naturel que, pour rendre possible une théorie de la science fidèle à la science en mouvement, Cavaillès proposât une philosophie du concept (plutôt qu'une philosophie de la conscience). On a d'autant plus épilogué sur cette formule qu'elle est demeurée programmatique dans l'œuvre tragiquement interrompue de Cavaillès. Nous ne prétendons pas donner la clef de l'énigme. D'autant moins que l'interprétation qui a détecté le spinozisme de Cavaillès (70) peut rendre compte, le terme lui-même mis à part, de la dialectique par laquelle l'idée et "l'idée de l'idée " manifestent leur "puissance génératrice"(71). Nous croyons simplement qu'on ne peut méconnaitre ce caractère conceptuel des mathématiques, si fortement souligné par l'école algébrique du début de ce siècle, si l'on veut avoir un modèle (au sens - logique exact du terme) de la dialectique des concepts chez Cavaillès; ou de la dialectique $d u$ concept, comme il l'écrit aussi (72), modifiant sensiblement l'expression et l'acculant à se figer, malgré lui, en catégorie de pensée. Pour Cavaillès, en effet, la propriété que les mathématiques sont seules à avoir de façon aussi entière est que le sens des théories est non pas dans les modes transitoires de leur actualisation, mais dans ce qu'il appelle "leur devenir conceptuel ». Tous les "contenus ", objets d'observation pour l'épistémologue et de manipulation pour le praticien : théorèmes, procédures de démonstration, opérations, propriétés, théories mêmes, sont traversés par un mouvement dans lequel la structure " se déroule et se démontre elle-même ". En mathématiques, c'est le devenir lui-même qui travaille pour l'intelligible - celui-ci étant moteur de celui-là -, pour l'essence contre l'accident, pour le nécessaire contre le contingent. Les concepts, schémas résultant

(70) G. G. Granger, Jean Cavaillès ou la montée vers Spinoza, Eludes philosophiques (juillet-décembre 1947), et G. Canguilhem, Vie et mort de Jean Cavaillès, Les Carnets de Baudasser (Ambialet (Tarn): Pierre Laleure (libraire-éditeur), 1984).

(71) Sur la logique..., op. cil., 32.

(72) Transfini et continu, Philosophie mathématique (Paris : Hermann, 1962), 273. 
de processus d'abstraction de différents ordres et s'exerçant selon diverses directions (73) sur des contenus plus ou moins complexes, sont des jalons dans le mouvement indéfini qui se ressource en eux pour un nouveau départ, vers de nouveaux enchaînements.

La "dialectique des concepts" nous semble donc s'identifier à l' "expérience " mathématique elle-même (74) telle que Cavaillès pouvait en refaire l'épreuve dans ces années 30 : marquée pardessus tout par une irrésistible montée vers l'abstraction.

Par un effet de redoublement ou de reflet, dont nous avons dit la raison d'être en commençant, Cavaillès propose une philosophie adaptée au développement effectif des mathématiques des structures et des concepts (75). Le modèle mathématique de la philosophie du concept c'est la dialectique objective des contenus mathématiques qui agissent et réagissent les uns sur les autres spontanément, dans un mouvement dont la conscience mathématicienne n'est qu'une détermination extrinsèque, au même titre que les données de culture ou de société. Mouvement de révision perpétuelle où chaque nouvelle " idéalisation " force l'intuition à arracher de nouvelles œillères tandis que le concept est sauvé par la mobilité de la structure : "Les procédés exigés par la solution d'un problème provoquent, dans l'actualisation même qui leur donne un sens, un tel changement d'éclairage qu'il faut déjà abandonner les notions qui forment leur structure " (76). Rien n'est moins synonyme d'arrêt ou de fixité que la structure. Non pas "uniforme " rigide mais " moment d'une dialectique créatrice ". Le processus de "thématisation " superpose indéfiniment les formes-principes aux formesopérations, les structures-concepts aux procédures idéalisées.

Mobilité des structures ou dialectique des concepts, ce sont là deux points de vue qui se correspondent. Pour la mathématique

(73) Comme nous l'avons vu en 5.1., des processus longitudinaux étagés s'enchevêtrent avec des processus transversaux également étagés.

(74) Jean Hyppolite l'avait bien reconnu dans ses objections à l'utilisation plurivoque, fort différente de celle de Cavaillès, du terme " dialectique " par Lautman. Voir le compte rendu de la séance du 4 février 1939 de la Société française de Philosophie, discussion, op. cit., n. 2, 28. Voir également la lettre de Cavaillès à son père du 28 avril 1938, dans G. Ferrières, op. cit., 119.

(75) Il taudrait relire le compte rendu des deux thèses de Cavaillès, que Charles Ehresmann fit en 1941, dans la Revue philosophique. Ehresmann souligne que les idées de Cavaillès répondaient " au sentiment intime de la plupart des mathématiciens de son temps $n$.

(76) Transfini et continu, op. cil., n. 72, 273-274. 
des structures quelle philosophie proposer sinon précisément une philosophie du concept?

Resterait à s'interroger sur la légitimité de l'extension prévue dès le départ par Cavaillès. Du champ particulier des mathématiques à un champ quelconque de pensée, le passage, s'il est possible, est-il sans problèmes ? Pour Cavaillès, la réponse ne fait pas de doute. En 1939, présentant la nouvelle collection qu'il vient de créer avec Raymond Aron dans la série des Actualités scientifiques et industrielles, Gavaillès écrivait :

"Ge que nous essaierons de dire a l'ambition d'être vrai et, comme tel, doit participer au destin de toute connaissance, s'informer en une architecture conceptuelle dont aussi bien la rigueur de structure que la puissance de changement se prêtent à communication : philosophia perennis sed in actione hominis manifesta" (77).

On reconnaît bien là, appliquées à la philosophie en général, les caractéristiques recueillies dans l'observation des mathématiques abstraites. Comme l'algébriste axiomaticien, le philosophe exploite l'expérience (78) d'un domaine particulier en vue de dégager une structure générale. Tout le problème, cependant, se trouve dans la justification de cette analogie.

CNRS.

Hourya Benis-Sinaceur.

(77) Cette présentation se trouve dans le fascicule 804 (1939) des Actualités scientifiques et industrielles, qui constituent le premier numéro de la collection "Essais philosophiques " publiés par Jean Cavaillès. Ce premier numéro est occupé par les Nouvelles Recherches sur la structure dialeclique des mathématiques de A. Lautman. I.a présentation est signée : Jean Cavaillès et Raymond Aron. La lettre a Lautman, du 6 décembre 1938 (ci-après, 125-126) nous apprend que Lautman y a participé : Cavaillès a "fusionné " le projet de Lautman avec le sien propre avant d'envoyer la synthèse à Aron. Documents S. L.

(78) Il faut donner son sens fort à ce mot. Voir les lettres à Lautman datées de septembre 1938 et du 7 novembre 1938, ci-après, 123-124. Voir la dêfinition de ce terme et les commentaires faits par Cavaillès dans le compte rendu de la séance du 4 février 1939 de la Société française de Philosophie, op. cit., n. 58, 9. 